\title{
Determinação de substâncias húmicas em diferentes tipos de compostos
}

As substâncias húmicas presentes na matéria orgânica do solo representam o compartimento de maior reatividade nos solos. Deste modo, mensurar as modificações que ocorrem na matéria orgânica do solo (MOS), a qual originam as substâncias húmicas (ácidos húmicos (AH); ácidos fúlvicos (AF) e humina (H)), auxilia no processo de diferenciação entre compostos orgânicos e vermicompostos que passaram por sistemas de compostagem. Diante disso, este estudo teve por objetivos determinar as substâncias húmicas presente em dois tipos de compostos orgânicos, sendo eles o substrato comercial e um substrato comercial adicionado a vermicompostagem de frutas e legumes, além de realizar um comparativo e definir as diferenças quanto os teores das frações das substâncias húmicas encontradas nos mesmos. A metodologia empregada para determinação das substâncias húmicas e seu fracionamento, análise espectroscópica de infravermelho, para caracterização dos diferentes compostos foi baseada em Antunes (2009). O composto comercial adicionado a vermicompostagem de frutas e legumes apresentou maior índice de humificação e percentual de humificação. Já o composto comercial isolado apresentou as maiores frações de AH e AF e maiores conteúdos de compostos aromáticos. A predominância de ácidos húmicos sobre os fúlvicos em ambos os compostos, indicou que ocorreram os processos de humificação adequada dos resíduos avaliados para os dois compostos.

Palavras-chave: Compostagem; Ácidos Húmicos; Ácidos Fúlvicos.

\section{Determination of humic substances in different types of compounds}

The humic substances present in soil organic matter represent the most reactive compartment in soils. Thus measuring the changes occurring in soil organic matte (SOM), which originate the humic substances (humic acids (AH), fulvic acids (AF) and humina (H)), helps in the process of differentiation between organic compounds and vermicompost that have passed through composting systems. Thus, the aim of this study was to determine the humic substances present in two types of organic compounds, being the commercial substrate and a commercial substrate added to the vermicomposting of fruits and vegetables, as well as to perform a comparative and to define the differences as the contents of the fractions of the humic substances found in them. The methodology used to determine the humic substances and their fractionation, the infrared spectroscopic analysis to characterize the different compounds was based on Antunes (2009). The commercial compound added to vermicomposting of fruits and vegetables had a higher humification index and humification percentage. On the other hand, the commercial compound presented the highest fractions of AH and FA and higher contents of aromatic compounds. The predominance of humic acids over fulvic in both compounds indicated that the processes of proper humification of the residues for the two compounds evaluated occurred.

Keywords: Composting; Humic Acids; Fulvic Acids.

Topic: Desenvolvimento, Sustentabilidade e Meio Ambiente

Reviewed anonymously in the process of blind peer
Received: $10 / 08 / 2018$

Approved: 24/09/2018
Carolina Faccio Demarco (iD)

Universidade Federal de Pelotas, Brasil http://lattes.cnpq.br/3166438553797256 http://orcid.org/0000-0002-3826-404X carol demarco@hotmail.com

\section{Thays França Afonso (iD)}

Universidade Federal de Pelotas, Brasil http://lattes.cnpq.br/4434140906417586 http://orcid.org/0000-0002-7803-7319 thaysafonso@hotmail.com

Samanta Tolentino Cecconello id Universidade Federal de Pelotas, Brasil http://lattes.cnpq.br/0667595394126577 http://orcid.org/0000-0001-5903-6241 satolentino@gmail.com

\author{
Natali Rodrigues dos Santos (iD \\ Universidade Federal de Pelotas, Brasil \\ http://lattes.cnpq.br/9930998387741584 \\ http://orcid.org/0000-0002-3377-3451 \\ natalisantosquimica@yahoo.com.br \\ Tatiana Nunes Diesel (iD) \\ Universidade Federal de Pelotas, Brasil \\ http://lattes.cnpq.br/0803839906522069 \\ http://orcid.org/0000-0002-9049-5718 \\ engambdiesel@gmail.com \\ Adriane Xavier Cortez \\ Universidade Federal de Pelotas, Brasil \\ http://lattes.cnpq.br/9177668159917514 \\ dridricortez@hotmail.com
}

\begin{abstract}
Robson Andreazza
Universidade Federal de Pelotas, Brasil http://lattes.cnpq.br/5706766977817721 robsonandreazza@yahoo.com.br

Maurizio Silveira Quadro (iD

Universidade Federal de Pelotas, Brasil http://lattes.cnpq.br/1749935262841216 http://orcid.org/0000-0001-8236-7479 mausq@hotmail.com
\end{abstract}

\section{Referencing this:}

DEMARCO, C. F.; AFONSO, T. F.; CECCONELLO, SS. T.; SANTOS, N. R.; DIESEL, T. N.; CORTEZ, A. X.; ANDREAZZA, R.; QUADRO, M. S.. Determinação de substâncias húmicas em diferentes tipos de compostos. Revista Ibero Americana de Ciências Ambientais, v.9, n.7, p.273-279, 2018. DOI: http://doi.org/10.6008/CBPC2179$\underline{6858.2018 .007 .0025}$ 


\section{INTRODUÇÃO}

Os solos são meios biológicos ricos em microrganismos e matéria orgânica (MO), que são alguns dos indicativos de qualidade dos solos. Os principais componentes da matéria orgânica do solo são as substâncias húmicas (SH), sendo os compostos orgânicos condensados que se dividem em ácidos fúlvicos (AF), ácidos húmicos (AH) e humina (HU) (ROSA et al., 2017; BALDOTTO et al., 2014).

As substâncias húmicas presentes na matéria orgânica do solo representam o compartimento de maior reatividade nos solos, ou seja, elas estão envolvidas na maior parte das reações químicas presentes nos solos (ROSA et al., 2017). Assim, avaliar os processos de degradação da matéria orgânica e a relação envolvida com a alteração dos resíduos orgânicos presentes no solo, que afetem a preservação ou re-síntese desses compostos nos mesmo, se torna uma ferramenta importante na avaliação de qualidade dos solos.

Deste modo, mensurar as modificações que ocorrem na matéria orgânica do solo (MOS), a qual originam as substâncias húmicas - colóides estáveis -, que representam cerca de 80-90\% da matéria orgânica do solo, se torna importante, já que, suas frações, bem como a alteração do carbono orgânico presente no solo podem influenciar na qualidade e manutenção dos mesmos (BALDOTTO et al., 2014; MENDONÇA et al., 2005). Silva et al. (2013) discute que os processos de humificação da matéria orgânica do solo são pouco entendidos, mediante alguns processos serem um tanto complexos, sendo assim o conhecimento a respeito dos indicadores das frações húmicas ainda é restrito.

Para tal, vem se estudando cada vez mais as frações da matéria orgânica do solo - as substâncias húmicas (SH), dentre essas frações, os ácidos fúlvicos são a parte solúvel que possuem compostos moleculares com massa molecular aparentemente inferior à dos ácidos húmicos, porém com maior conteúdo de grupos funcionais ácidos. Os ácidos húmicos são compostos escuros, quimicamente complexos, o que evidencia a alta capacidade de troca catiônica, enquanto, os ácidos fúlvicos representam as frações claras e quimicamente constituídas por polissacarídeos, aminoácidos, compostos fenólicos, além de possuírem capacidade redutora nos solos formando complexos de $\mathrm{Fe}, \mathrm{Cu}, \mathrm{Ca}$ e Mg (IHSS, 2017). Por fim, a fração humificada equivale às substâncias que não são extraídas do solo, estando estreitamente ligadas à fração mineral do solo, denominada humina. A classificação dessas frações das substâncias húmicas presentes nos solos são então baseadas na capacidade de estas se solubilizarem em meios alcalinos ou ácidos (BALDOTTO et al., 2014).

Nos solos, a presença de resíduos orgânicos (materiais biodegradáveis) colaboram na retenção de umidade fornecendo macronutrientes e micronutrientes às plantas, além de melhorar a textura do solo reduzindo a entrada de $\mathrm{CO}_{2}$ na atmosfera. A reciclagem desses resíduos através dos processos de compostagem tem contribuído para ciclagem de carbono e nutrientes no solo (MELO et al., 2008; LOUREIRO et al., 2007). Desde modo existe um grande estímulo e interesse pela variabilidade na produção de vermicomposto e compostos orgânicos maturados, frutos do processo de compostagem, em função da sua capacidade estabilizar a matéria orgânica (MO) em substâncias húmicas, que são compostos muito mais estáveis (VALENTE et al., 2009). 
Segundo Cotta et al. (2015) nos processos de vermicompostagem e compostagem, nota-se a diminuição no teor de carbono orgânico e o aumento do teor de ácido húmico, relacionado aos mecanismo de humificação, possibilitando um aumento da matéria orgânica, disponibilização de nutrientes tanto para o solo quanto para as planta, além de melhorar a CTC do solo.

Diante do exposto, este estudo teve como objetivos determinar as substâncias húmicas presente em dois tipos de compostos orgânicos: sendo eles o substrato comercial e um substrato comercial adicionado a vermicompostagem de frutas e legumes, além de realizar um comparativo e definir as diferenças quanto os teores das frações das substâncias húmicas encontradas nos mesmos.

\section{MATERIAIS E MÉTODOS}

Foram analisados dois compostos, um na forma comercial isolada, denominado Composto 1 (principais componentes: tanino, esterco de galinha, calcário, farinha de osso e terra preta) e o Composto 2 (Composto 1 adicionado vermicompostagem de cascas de frutas, verduras e legumes e este maturado em 50 dias). Os experimentos foram realizados no laboratório de Química Ambiental (LQA) do Centro de Engenharias, da Universidade Federal de Pelotas.

Para o fracionamento químico da matéria orgânica pressentes nos Compostos 1 e 2, em ácidos húmicos (AH) e ácidos fúlvicos (AF), baseou-se na capacidade de estes se solubilizarem em meio ácido e básico (DICK et al., 1997). Retirou-se $3 \mathrm{~g}$ de amostra tanto do Composto 1 quanto do Composto 2 (trituradas e peneiradas), secas a uma temperatura $65^{\circ} \mathrm{C}$ por 24 horas, anotando-se o peso final das mesmas. Adicionouse $40 \mathrm{ml}$ de $0,5 \mathrm{~mol} \mathrm{~L}^{-1} \mathrm{HCl}$ aos compostos 1 e 2, posteriormente feita a agitação lenta das amostras em (4080 rpm proporcionado movimentos laterais) por 2 horas; após as amostras foram centrifugadas 15 minutos a $2500 \mathrm{rpm}$.

Separou-se o sobrenadante, contendo a fração não húmica $(\mathrm{NH})$, após o processo de centrifugação (15 min a $2.500 \mathrm{rpm}$ ) e mediu-se o volume total. Ao resíduo sólido precipitado no fundo do frasco foram adicionados $30 \mathrm{ml}$ de $0,5 \mathrm{~mol} \mathrm{~L}^{-1} \mathrm{NaOH}$, procedeu-se à agitação por 3 horas e à centrifugação (15 minutos a $2.500 \mathrm{rpm}$ ), repetindo-se esse procedimento até o sobrenadante ficar claro. Em cada extração, filtrou-se o sobrenadante e transferiu-o para um frasco coletor, determinando o volume final para $500 \mathrm{ml}$ desse sobrenadante que contém as substâncias húmicas solúveis (SHS=AH+AF).

Posteriormente, retirou-se uma alíquota de $20 \mathrm{ml}$ para análise de carbono (C) e o restante acidificado a pH 2,0 com 0,5 mol L-1 $\mathrm{HCl}$. Após $24 \mathrm{~h}$ em repouso em pH ácido, separou-se por centrifugação os $\mathrm{AF}$ solúveis dos AH insolúveis. Em seguida determinou espectroscopicamente em absorbância de $580 \mathrm{~nm}$ o (C) referente aos extratos das frações $\mathrm{NH}, \mathrm{AF}$ e $\mathrm{SH}$.

Utilizou-se uma curva padrão de D-glucose anidra de 0 a 250 mgC. $\mathrm{L}^{-1}$ para quantificação do C, após a reação com solução ácida de dicromato de potássio $1,25 \mathrm{~mol} \mathrm{~L}^{-1}$, durante $4 \mathrm{~h}$ a 60 으. Corrigiu-se as concentrações de C para o método Walkley-Black pela Equação 1, obtida em estudo de correlação entre o método Walkley-Black e o espectrofotométrico $(r=0,993)$ com 6 amostras aleatórias do experimento para a 
equação da reta y $=0.0013 x+0.0105$ encontrada, e o C foi determinado por ambos os métodos (ANTUNES, 2009).

Através da Equação 2, determinou-se o teor de C na forma de AH. A Equação 3 permitiu verificar o teor de C na fração insolúvel (FI). Calculou-se pela Equação 4 a distribuição de cada fração como percentual do C para os índices de humificação e o percentual de AH (Chefetz et al., 1996). Determinou-se para os ácidos fúlvicos a razão E4/E6 (razão entre as absorbâncias lidas em 465 e 665 nm) representando o grau de humificação da matéria orgânica desses compostos 1 e 2.

$$
\begin{gathered}
\text { CWalkley Black }=(0,664 \times \text { C espectrofotometrico })-10,234 \quad \text { Eq. } 1 \\
\text { CAH }=\mathrm{CSH}-\mathrm{CAF} \text { Eq. } 2 \\
\mathrm{CFI}=\mathrm{COT}-(\mathrm{CNH}+\mathrm{CSH}) \quad \text { Eq. } 3 \\
\mathrm{IH}=\frac{\mathrm{CAH}}{\mathrm{CSH}} \times 100 \quad \text { Eq. } 4
\end{gathered}
$$

\section{RESULTADOS E DISCUSSÃO}

\section{Carbono orgânico total (COT)}

Os teores de carbono orgânico total (COT) inicial foram de 211,41 g kg-1 no Composto 1 (Comercial) e 378, $51 \mathrm{~g} \mathrm{~kg}^{-1}$ no Composto 2 (Comercial- vermicomposto) (Tabela 1). Antunes et al. (2015) analisando o substrato de vermicompostagem de restos de frutas e vegetais, obteve um valor de $365,50 \mathrm{~g} \mathrm{~kg}^{-1} \mathrm{de} \mathrm{COT} \mathrm{em}$ amostras de 42 dias, o que assemelha-se com o encontrado neste trabalho para o Composto 2 (composto

\begin{tabular}{|c|c|c|}
\hline Parâmetros & Composto 1 & Composto 2 \\
\hline & \multicolumn{2}{|c|}{--------------g kg ${ }^{-1}$} \\
\hline COT & 211,41 & 378,51 \\
\hline AF & 4,17 & 1,06 \\
\hline $\mathrm{AH}$ & 65,80 & 44,43 \\
\hline SHS & 69,96 & 45,49 \\
\hline $\mathrm{FI}$ & 140,31 & 332,74 \\
\hline
\end{tabular}
comercial adicionado ao vermicomposto de frutas, legumes e vegetais).

Tabela 1: Teores de carbono orgânico total (COT), frações dos ácidos húmicos (AH), ácidos fúlvicos (AF), substâncias húmicas solúveis (SHS) e fração insolúvel (FI) dos compostos analisados.

Pode-se justificar o Composto 1 com COT inferior ao Composto 2 devido ao fato de ser um composto pronto, e deste modo ter finalizado todos os estágios da compostagem e humificação, corroborando estudos de Dores-Silva et al. (2013), onde o COT diminuiu após os processos de compostagem e vermicompostagem para todas as matrizes estudadas. Antunes et al. (2015) também obtendo uma redução significativa nos teores de COT para diferentes compostos (esterco bovino, esterco ovino, restos de alimentos de restaurantes e restos de frutas e vegetais), submetidos a vermicompostagem, justifica esses teores pela mineralização do material ocorrida durante o processo, que ocasiona perdas de $\mathrm{C}$ na forma de $\mathrm{CO}_{2}$.

Quando comparado os valores de COT com a fração inorgânica, observa-se que na amostra com maior fração de COT apresenta maior fração insolúvel. Essa relação também foi identificada por Antunes et al. (2015), onde os compostos estudados também apresentaram a fração insolúvel mais elevada nos compostos com maior COT. 


\section{Ácidos fúlvicos (AF) e ácidos húmicos (AH)}

Em relação ao teor de $C$ na fração $A F$ (Figura 1), o valor obtido para o Composto $1\left(4,17 \mathrm{~g} \mathrm{~kg}^{-1}\right)$ foi superior ao teor do Composto $2\left(1,06 \mathrm{~g} \mathrm{~kg}^{-1}\right)$. Ambos os valores de AF foram inferiores aos teores de $\mathrm{AH}$ (65,80 $\mathrm{g} \mathrm{kg}^{-1}$ e 44,43 $\mathrm{g} \mathrm{kg}^{-1}$ no Composto 1 e 2 respectivamente), corroborando a afirmativa de Silva et al. (2009) de que a predominância de ácidos húmicos sobre os fúlvicos no final da compostagem é um indicativo de humificação adequada dos resíduos avaliados, mesmo o Composto 2 não estando em seu estágio final de maturação.

Os teores da fração de Substâncias Húmicas Solúveis (SHS) foi superior no Composto 1, tendo em vista que o valor de SHS é a soma das frações de AF e AH, ao contrário do encontrado por Cotta et al. (2015), os quais demonstraram em seus estudos comparativos entre compostagem e vermicompostagem aplicados para três tratamentos (serragem e resíduo animal; resíduos verdes; resíduos verdes e resíduos animais), que ocorreu maior teor de substâncias húmicas no vermicomposto para todos os tratamentos. Destaca-se que o vermicomposto é um produto normalmente mais estável (WANG et al., 2014). A Figura 1 permite visualizar as diferentes frações nos compostos.

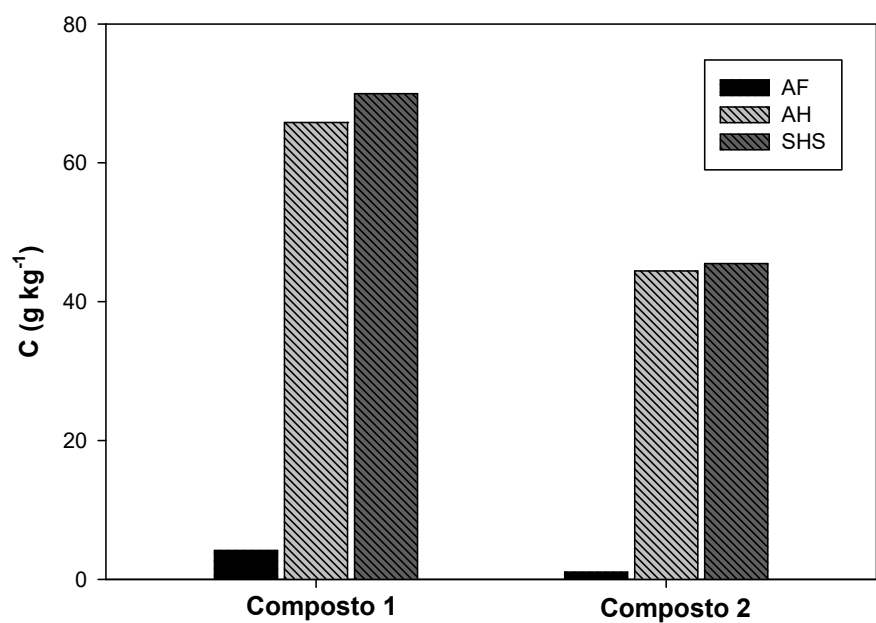

Figura 1: Distribuição das frações da matéria orgânica: AF (ácidos fúlvicos), AH (ácidos húmicos) e SHS (substâncias húmicas solúveis) nos compostos analisados.

\section{Índice de humificação (IH) e percentual de humificação (PH)}

O processo de compostagem e vermicompostagem em geral é acompanhado pelo aumento do índice de humificação $(\mathrm{IH})$ e do percentual de humificação $(\mathrm{PH})$, onde os maiores valores indicam um maior grau polimerização, e consequentemente maior maturação (ANTUNES et al., 2015).

Tabela 2: Índice de humificação $(\mathrm{IH})$, percentual de humificação $(\mathrm{PH})$ e relação E4/E6 ácidos fúlvicos, para os dos compostos analisados.

\begin{tabular}{|l|l|l|}
\hline Parâmetros & Composto 1 & Composto 2 \\
\hline $\mathrm{HI}$ & 15,79 & 41,88 \\
\hline $\mathrm{PH}(\%)$ & 94,07 & 97,75 \\
\hline E4/E6 SH & 2.9 & 4.23 \\
\hline E4/E6 AF & 8,43 & 8,84 \\
\hline
\end{tabular}

Na Tabela 2, pode-se verificar que o Composto 2 apresenta o maior índice de humificação $(41,88)$ e percentual de humificação (97,75\%), quando comparado ao Composto 1 (15,79 e 94,07\%). Isso indica que o 
grau de maturação do Composto 2 é maior que o do composto 1. Conforme demonstrado por Chefetz et al., (1996) em seus estudos, o processo de compostagem é seguido pelo aumento da razão de humificação, e do percentual de ácidos húmicos, ou seja, neste caso o composto comercial acrescido de vermicomposto de resíduos de origem vegetal apresentou estágios mais avançados de maturação.

\section{E4/E6 AF}

A relação E4/E6 para AF (Tabela 2) foram similares no Composto $1(8,43)$ e Composto $2(8,84)$. Ambos os compostos estão dentro do proposto estabelecido por Kononova (1966), onde a relação E4/E6 para AF apresentam valores entre 6,0 e 8,5. A proposta do autor teve como base os valores da relação E4/E6 de solos de clima temperado. A autora observou valores para os AH variando de 3,5 (A chernozêmico) a 5,0 (B espódico) e para os AF de 6,0 a 8,5, para os mesmos horizontes. Para solos de clima subtropical e tropical os valores dessa relação podem variar desde menores que 3,5, a maiores que 5,0 , para os AH e maiores que 8,5, para os AF. Assim, quanto menor a razão E4/E6, maior é a aromaticidade presente nos compostos segundo Chen et al. (1997). Desta forma, nota-se que o Composto 1 apresenta razões E4/E6 inferiores tanto para SH quanto $\mathrm{AF}$ ao Composto 2, demonstrando que o seu conteúdo de elementos aromáticos é bem maior.

\section{CONCLUSÕES}

Apesar de o composto comercial adicionado a vermicompostagem de frutas, verduras e legumes com 50 dias de maturação (Composto 2) apresentar maior índice de humificação e percentual de humificação, o composto comercial isolado (Composto 1) teve as maiores frações de $\mathrm{AH}$ e $\mathrm{AF}$ e maiores conteúdos de compostos aromáticos. A predominância de ácidos húmicos sobre os fúlvicos em ambos os compostos indicou que ocorreram os processos de humificação adequada dos resíduos avaliados nos dois compostos. Entretanto, recomenda-se a avaliação do fracionamento da matéria orgânica ao longo do tempo de maturação tanto da compostagem quanto da vermicompostagem, permitindo assim verificar as transformações químicas inerentes ao processo de estabilização dos compostos orgânicos.

\section{REFERÊNCIAS}

ANTUNES, R. M.; CASTILHOS, R. M. V.; CASTILHOS, D. D., LEAL, O. A., DICK, D. P.; ANDREAZZA, R.. Transformações químicas dos ácidos húmicos durante o processo de vermicompostagem de resíduos orgânicos. Engenharia Sanitária e Ambiental, v.20, n.4, p.699-708, 2015. DOI: http://doi.org/10.1590/S1413-41522015020040114466

ANTUNES, R. M.. Humificação de resíduos orgânicos durante a vermicompostagem e seu efeito nos atributos químicos do solo e no crescimento inicial de acácia negra (Acacia nearnsii). Tese (Doutorado em agronomia) Universidade Federal de Pelotas, Pelotas, 2009.

BALDOTTO, M. A.; BALDOTTO, L. E. B.. Ácidos húmicos. Revista Ceres, v.61, p.856-881, 2014. DOI http://doi.org/10.1590/0034-737X201461000011
CHEFETZ, B.; HATCHER, P.; HADAR, Y.; CHEN, Y.. Chemical and biological characterization of organic matter during composting of municipal solid waste. Journal of Environmental Quality, v.25, p.776-785, 1996. DOI: http://doi.org/10.2134/jeq1996.00472425002500040018x

CHEN, Y.; SCHNITZER, M.. Information provided on humic substances by E4/E6 ratios. Soil Science of America Journal, v.41, p.352-358, 1977. DOI:

http://doi.org/10.2136/sssaj1977.03615995004100020037x

COTTA, J. A. O.; CARVALHO, N. L. C.; BRUM, T. S.; REZENDE, M. O.. Compostagem versus vermicompostagem: comparação das técnicas utilizando resíduos vegetais, esterco bovino e serragem. Engenharia Sanitária e Ambiental, v.20, n.1, p.65-78, 2015. DOI: http://doi.org/10.1590/S1413-41522015020000111864 
DICK, D. P.; NOVOTNY, E. H.; DIECKOW, J.; BAYER, C..

Química da matéria orgânica do solo. In: MELLO, V. F.;

ALLEONI, L. R. F.. Química e mineralogia do solo. Viçosa: SBCS, 2009. p.1-68.

DICK, D. P.; DICK, Y. P.; SILVA, L. F.; ESSIG, C.; ZORTEA, R. B.. Caracterização de produtos de decomposição de lodos orgânicos em diferentes tempos e meio de digestão. Revista Brasileira de Ciência do Solo, n.21, p.1-8, 1997.

DORES-SILVA, P. R.; LANDGRAF, M. D.; REZENDE, M. O. O.. Processo de estabilização de resíduos orgânicos: vermicompostagem versus compostagem. Químicaii Nova, v.36, n.5, p.640-645, 2013. DOI:

http://doi.org/10.1590/S0100-40422013000500005

IHSS. International Humic Substances Society Products. Natural Organic Matter Research. What are Humic Substances?. 2017.

KONONOVA, M. M.. Soil organic matter. 2 ed. Oxford: Pergamon Press, 1966.

MENDONÇA, E. S.; MATOS, E. S.. Matéria orgânica do solo: métodos de análises. Viçosa: UFV, 2005.

ROSA, D. M.; NOBREGA, L. H. P.; MAULI, M. M.; LIMA, G. P.; PACHECO, F. P.. Substâncias húmicas do solo cultivado com plantas de cobertura em rotação com milho e soja. Revista Ciência Agronômica, v.48, n.2, p.221-230, 2017. DOI: http://doi.org/10.5935/1806-6690.20170026
SILVA, F. A. M.; LOPEZ, F. G.; VILLAS BOAS, R. L.; SILVA, R. B. Transformação da matéria orgânica em substâncias húmicas durante a compostagem de resíduos vegetais. Rev. Bras. de Agroecologia, v.4, n.1, p.59-66, 2009.

SILVA, A. C.; SILVA, V. E.; CAMARGO, B. P. C.; PEREIRA, R. C. B.; BOTELHO, U. M.; TORRADO, A. M. M.; VDAL, P. .

Composição lignocelulósica e isótopica da vegetação e da matéria orgânica do solo de uma turfeira tropical substâncias húmicas e processos de humificação. Revista Brasileira de Ciência do Solo, v.37, n.1, p.134-144, 2013. DOI: http://doi.org/10.1590/S0100-06832013000100014

SILVA, I. R.; MENDONÇA, E. S.. Matéria orgânica do solo. In: NOVAIS, R. F.; ALVAREZ, V. H.; BARROS, N. F.; FONTES, R. L. F.; CANTARUTTI, R. B.; NEVES, J. C. L.. Fertilidade do solo. Viçosa: Sociedade Brasileira de Ciência do Solo, 2007. p.275374

VALENTE, B. S.; XAVIER, E. G.; MORSELLI, T. B. G. A.; JAHNKE, D. S., BRUM JR., B. S.; CABRERA, B. R.; MORAES, P. O.; LOPES, D. C. N.. Fatores que afetam o desenvolvimento da compostagem de resíduos orgânicos. Arquivos de Zootecnia, v.58, p.59-85, 2009.

WANG, K.; LI, X.; HE, C.; CHEN, C.; BAI, J.; REN, N.; WANG, J. Transformation of dissolved organic matters in swine, cow and chicken manures during composting. Bioresource Technology, v.168, p.222-228, 2014. DOI: http://doi.org/10.1016/j.biortech.2014.03.129

A CBPC - Companhia Brasileira de Produção Científica (CNPJ: 11.221.422/0001-03) detém os direitos materiais desta publicação. Os direitos referem-se à publicação do trabalho em qualquer parte do mundo, incluindo os direitos às renovaç̃ões, expansões e disseminações da contribuiç̃o, bem como outros direitos subsidiários. Todos os trabalhos publicados eletronicamente poderão posteriormente ser publicados em coletâneas impressas sob coordenação da Sustenere Publishing, da Companhia Brasileira de Produção Científica e seus parceiros autorizados. Os (as) autores (as) preservam os direitos autorais, mas não têm permissão para a publicação da contribuição em outro meio, impresso ou digital, em português ou em tradução. 